\title{
Plataforma Robocode como Ferramenta Lúdica de Ensino de Programação de Computadores- Extensão Universitária em Escolas Públicas de Minas Gerais
}

\author{
Laurence Rodrigues do Amaral ${ }^{1}$, Gláucia Braga e Silva ${ }^{2}$, Eliana Pantaleão ${ }^{1}$ \\ ${ }^{1}$ Faculdade de Computação \\ Universidade Federal de Uberlândia (UFU) - Patos de Minas, MG - Brasil \\ ${ }^{2}$ Instituto de Ciências Exatas e Tecnológicas \\ Universidade Federal de Viçosa (UFV) - Florestal, MG - Brasil \\ \{laurence, epantaleao\}@ufu.br, glaucia@ufv.br
}

\begin{abstract}
This paper describes the use of the Robocode platform for algorithms and programming languages teaching in high school. The experience was developed with the help of undergraduate students that had already concluded courses on computer programming and acted as tutors and co-advisors of the younger students. Robocode environment was used as a support tool, using a playful learning strategy, providing an early contact of the high-school students with Java programming language. Besides programming learning, results include higher motivation in high school students to learn computer programming and the development of leadership abilities in undergraduate students, that came from tutoring and co-advising.
\end{abstract}

Resumo. Esse artigo descreve a experiência do uso da ferramenta Robocode no ensino de algoritmos e programação de computadores para alunos do Ensino Médio. O estudo envolveu também alunos de graduação que já haviam cursado disciplinas de programação de computadores e que atuaram como tutores $e$ coorientadores dos alunos. O ambiente Robocode foi utilizado como ferramenta de apoio ao ensino de algoritmos e linguagens de programação, com base em uma estratégia lúdica de ensino, sugerindo um contato mais precoce com a linguagem de programação Java, ainda no Ensino Médio, a fim de estimular o raciocínio lógico-matemático dos envolvidos de forma rápida e divertida. Além do aprendizado de programação de computadores pelos alunos, os resultados observados incluem o aumento do interesse pela programação nos alunos do Ensino Médio e o desenvolvimento da capacidade de liderança dos alunos de graduação, proveniente da experiência em coorientar os alunos aprendizes.

\section{Introdução}

Nos últimos anos, observam-se o esforço e o interesse na pesquisa e no desenvolvimento de recursos tecnológicos voltados a apoiar o processo de ensino-aprendizagem. Em especial, destacam-se ferramentas computacionais que oferecem ambientes lúdicos e interativos, capazes de atrair a atenção de crianças e jovens, aumentando o interesse e favorecendo a aprendizagem de conteúdos escolares. 
Acredita-se que o conhecimento das tecnologias disponíveis atualmente, em especial os jogos, seja fundamental para o trabalho educacional transformador e de qualidade [Leite et al. 2003]. Ao aliar conteúdo educacional à diversão e ao encantamento de um jogo, tem-se uma excelente ferramenta educacional. No entanto, o desafio é tornar o processo de aprendizagem algo imperceptível durante a brincadeira, o que exige cuidadoso estudo do design do jogo e das técnicas pedagógicas a serem aplicadas [Maike et al. 2011]. Além disso, as atividades devem ser planejadas e trabalhadas de uma forma crítica, que possibilite a aprendizagem de uma maneira significativa ao aprendiz [Pietruchinski et al. 2011].

O ensino de algoritmos e linguagens de programação busca nas Ciências Exatas seu pilar de sustentação, pois disciplinas nessa área despertam o raciocínio lógicomatemático para resolução de problemas [Santos and Costa 2006]. Por esse motivo, estuda-se hoje a inclusão do ensino de programação de computadores o mais cedo possível, ou seja, no Ensino Médio e até mesmo no Ensino Fundamental. Nos EUA, a Association for Computing Machinery (ACM), juntamente com outras instituições, propõe iniciativas de levar Computação ao Ensino Médio: a iniciativa $C S 10 K$ objetiva levar 10 mil professores de Computação ao Ensino Médio distribuídos em 10 mil escolas [Astrachan and Briggs 2012]. Uma outra iniciativa relacionada é a APCS Principles que pretende validar créditos do Ensino Médio no Ensino Superior. O primeiro curso seguindo a formatação estabelecida será oferecido em 2016 [Washington University 2015]. No Brasil, merece destaque o Scalable Game Design Brasil (SGD-Br) [PUC-Rio/SERG 2013], um projeto de pesquisa associado ao Scalable Game Design da Universidade do Colorado. O SGD-Br buscou desenvolver tecnologias para o ensino-aprendizado de raciocínio computacional para alunos do Ensino Fundamental do Estado do Rio de Janeiro, tal como a ferramenta PoliFacets [PUC-Rio/SERG 2015]. A pesquisa brasileira é realizada pelo Grupo de Pesquisa em Engenharia Semiótica (SERG) da PUC-Rio e pelo Laboratório de Documentação Ativa e Design Inteligente (ADDLabs) da Universidade Federal Fluminense, com o apoio da AgentSheets, Inc.

Esse artigo endereça o uso do ambiente Robocode como ferramenta de apoio ao ensino de algoritmos e linguagens de programação. A abordagem proposta baseia-se em uma estratégia lúdica de ensino, que sugere um contato mais precoce com a linguagem de programação Java, ainda no Ensino Médio, a fim de estimular o raciocínio lógicomatemático dos envolvidos de forma rápida e divertida. Com essa experiência, buscou-se:

1. Investigar o potencial didático-pedagógico do ambiente Robocode;

2. Estimular o raciocínio lógico-matemático dos alunos envolvidos;

3. Apoiar o ensino de Matemática no Ensino Médio;

4. Introduzir programação de computadores para alunos de Ensino Médio;

5. Integrar Universidade e Escola Pública; e

6. Captar alunos para a Universidade.

\section{Trabalhos Relacionados}

Essa seção destaca alguns trabalhos relacionados ao desenvolvimento e ao uso de recursos computacionais como mecanismos de apoio ao processo de ensino-aprendizagem. Nesse cenário, destaca-se o uso de jogos como uma estratégia lúdica para atrair o interesse dos alunos. Um exemplo é a plataforma Alice desenvolvida na universidade Carnegie Mellon 
[Carnegie Mellon University 2015]. Alice corresponde a um ambiente de programação 3D que facilita a criação de animações utilizadas para contar uma estória, para jogar um jogo interativo ou para compartilhar um vídeo na web. Trata-se de uma ferramenta de ensino livre projetada para iniciar o estudante no universo da programação orientada a objetos. Morati e colaboradores [Roberto G. Morati Jr. 2012] apresentam o desenvolvimento do jogo Alice no Labirinto das Decisões, implementado por meio do ambiente de desenvolvimento Alice, que coloca o jogador em situações de tomada de decisão que podem ajudá-lo a desenvolver habilidades em situações no mundo real.

Destaca-se também o estudo realizado com professores de uma Escola Pública de Ensino Fundamental de Campinas com o intuito de levantar requisitos para a concepção de uma ferramenta que permita aos professores criarem aventuras de RPG pedagógicas [Maike et al. 2011].

Batista Júnior e Santos Filho [Batista Junior and Santos Filho 2011] propõem o uso de ambientes virtuais como auxiliadores no ensino de Física em escolas públicas do Ensino Médio. Também relacionado à melhoria no ensino de Física, outro trabalho que merece destaque aborda o uso de Realidade Aumentada na construção de uma ferramenta lúdica de apoio ao ensino de Física [Viegas et al. 2012]. O sistema foi desenvolvido para utilização no Centro de Ciências da Universidade Federal de Juiz de Fora, que oferece atividades, cursos e programas de formação continuada para professores da Educação Básica.

Com relação ao uso de jogos para auxiliar o ensino de algoritmos e programação de computadores, Vahldick e Mattos [Vahldick and Mattos 2008] propuseram um framework em que os alunos programam, em Java, a inteligência de um robô que vive num mundo bidimensional em companhia de outros elementos, como paredes, alienígenas e tesouros. A ferramenta Furbot foi utilizada no primeiro semestre do curso de Ciência da Computação e conseguiu aumentar a motivação dos alunos no aprendizado de programação. Oliveira e colaboradores [de Oliveira et al. 2014] apresentaram o jogo GrubiBots Educacional, para o ensino de algoritmos no Ensino Fundamental. O jogo não necessita do desenvolvimento de códigos de programação e pode ser usado para resolver problemas de diversas áreas de conhecimento.

\section{A ferramenta Robocode}

O Robocode [SourceForge 2015] foi desenvolvido pela AlphaWorks, divisão pertencente à IBM, com o objetivo de divulgar novas tecnologias de desenvolvimento. Trata-se de um ambiente de simulação de batalha entre robôs, em que se joga programando em Java. $\mathrm{O}$ objetivo principal de um jogador é utilizar as classes bases disponibilizadas pelo ambiente na criação do seu próprio robô e colocá-lo em batalha com outros robôs criados por outros desenvolvedores. Para programar um robô, estende-se uma classe da API do Robocode, podendo-se programar robôs guerreiros dos mais variados tipos. Os robôs possuem radares, podem atirar, batem uns nos outros e movimentam-se livremente em qualquer ângulo. O ambiente contém um editor de código-fonte próprio para programar os robôs (Figura 1) e um espaço para criar uma arena e configurar a batalha adicionando os robôs.

A interatividade com o usuário é programada através do tratamento de eventos do mouse e do teclado. Além disso, existem eventos para avisar ao robô quando ele acerta um tiro ou é acertado, quando ele é eliminado da batalha, ou quando ela é finalizada. 


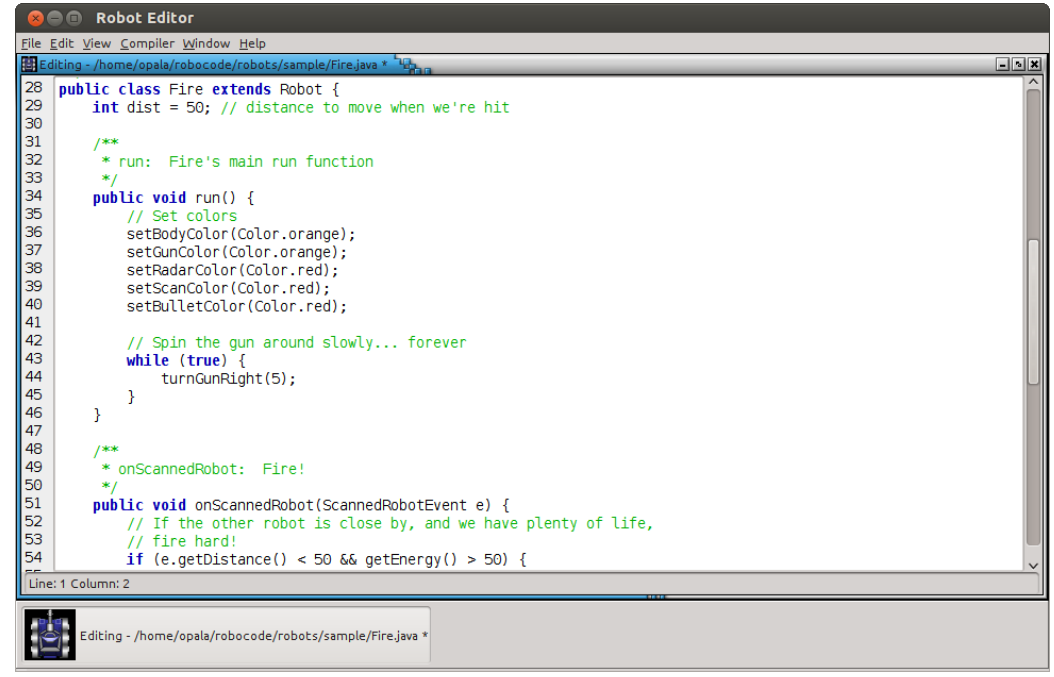

Figura 1. Editor de código-fonte do ambiente Robocode

A lógica dos robôs no Robocode está concentrada em métodos, que definem as ações e os movimentos dos robôs. Além disso, a movimentação dos robôs e algumas ações, como atirar em outros robôs, baseiam-se na definição de ângulos pré-especificados, ou seja, requer a aplicação direta de conceitos matemáticos. Como pode ser observado na Figura 2, pode-se alterar: a direção de rotação do robô e do canhão, que podem ser movimentados para a direita ou esquerda, individualmente ou em conjunto; direção de movimentação, podendo ir para frente ou para trás; além das características relacionadas à bala, podendo alterar seu poder de fogo, direção e velocidade.

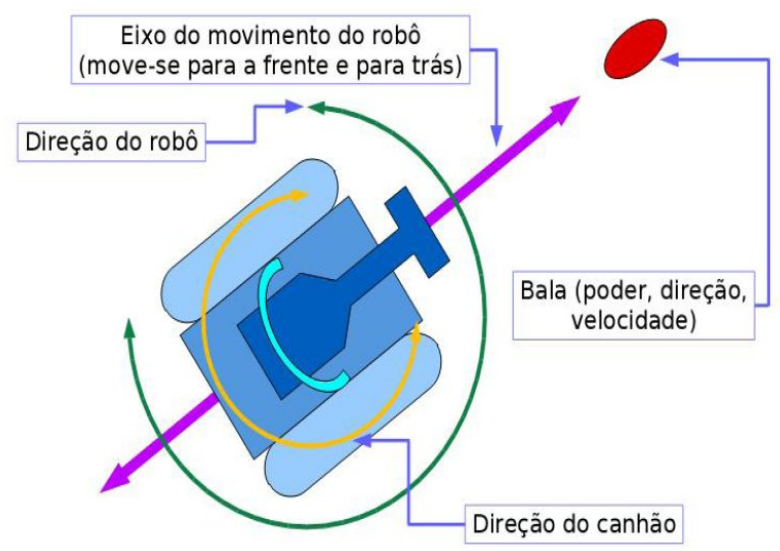

Figura 2. Características relacionadas ao movimento do robô

Cada robô é uma entidade que controla o seu próprio ciclo de vida. Quando uma batalha começa, um método específico, contendo a inteligência de cada robô, é disparado, e enquanto esse método estiver executando, provavelmente o robô está andando e/ou atirando [Vahldick and Mattos 2008]. A Figura 3 ilustra uma batalha realizada no Robocode, onde estão representados cinco robôs, chamados: Crazy, Target, Tracker, TrackFire e Fire. Esses robôs são robôs-modelo fornecidos pela plataforma Robocode. 


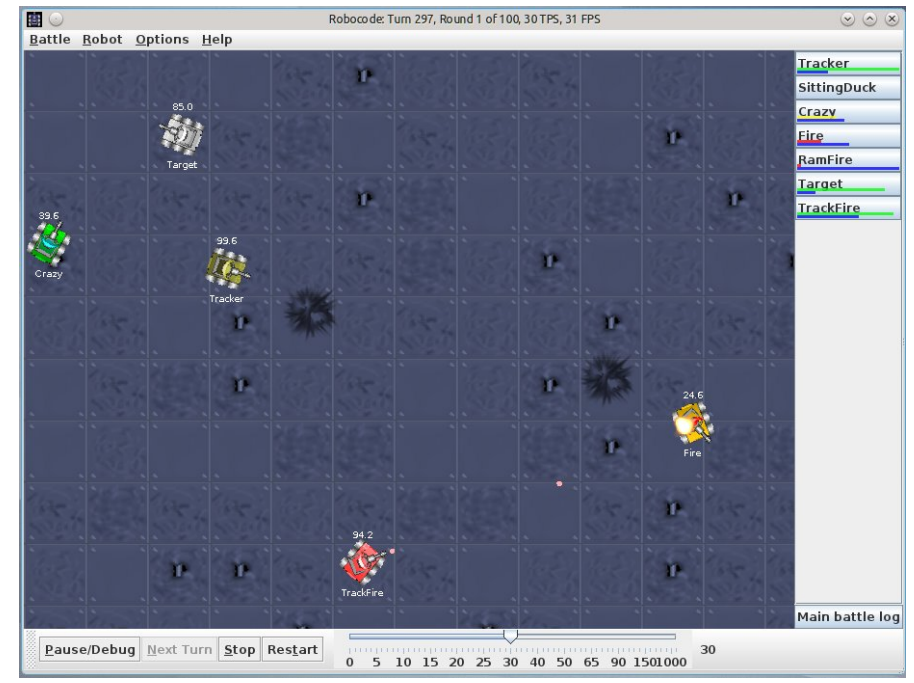

Figura 3. Robocode: Batalha entre robôs

\section{Estudo de Caso: Explorando o potencial do Robocode como ferramenta de ensino}

Esse artigo apresenta a experiência realizada em projetos de iniciação científica júnior (PIBIC-JR) e projetos de extensão universtária (PEIC) no período de 2012 a 2014. Os projetos foram realizados em conjunto pela Universidade Federal de Uberlândia (UFU) e escolas públicas da cidade de Patos de Minas/MG. Em cada versão, os alunos de graduação selecionados estavam entre o terceiro e o sexto período de Engenharia Eletrônica e de Telecomunicações e já haviam concluído com êxito disciplinas de programação de computadores. Já os alunos do PIBIC-JR estavam cursando o segundo ano do Ensino Médio. Os recursos financeiros restringiram-se às bolsas concedidas.

\subsection{Metodologia de Desenvolvimento do Estudo de Caso}

As atividades desenvolvidas no Estudo de Caso foram agrupadas em 5 etapas, conforme ilustra a Figura 4. Tais atividades totalizaram uma carga-horária de 232 horas.

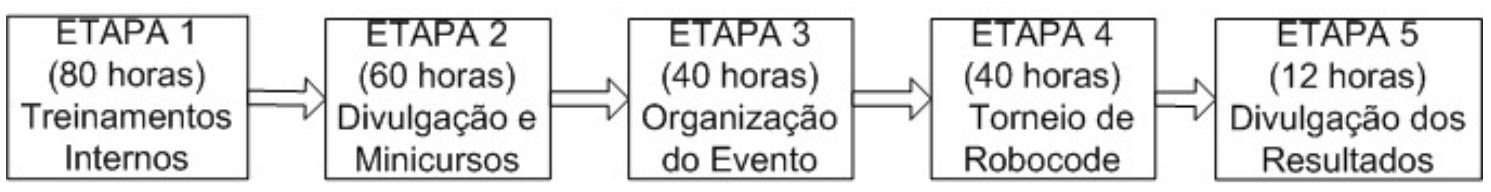

Figura 4. Etapas de Execução do Estudo de Caso

$\mathrm{Na}$ primeira etapa, todos os participantes receberam treinamentos envolvendo os seguintes tópicos: Introdução à Lógica Matemática e à Lógica de Programação; Algoritmos: Teoria e prática; Linguagem de programação Java; Orientação a Objetos em Java; e Ambiente Robocode. Os bolsistas do PIBIC-JR receberam um treinamento mais aprofundado nos tópicos de algoritmos e linguagem de programação Java. Esse treinamento foi realizado com o auxílio do ambiente Robocode, permitindo-se aplicar internamente a estratégia de ensino baseada em jogos educacionais. Esse treinamento foi realizado por alunos de graduação do curso de Engenharia Eletrônica e de Telecomunicações, chamados de tutores. 
Já nessa etapa, foi possível perceber o interesse dos jovens pelo ambiente Robocode, o que tornou o processo de aprendizagem mais atrativo aos olhos dos adolescentes. Além disso, o foco no raciocínio lógico-matemático é despertado intuitivamente pois o estudante fica motivado pelo desafio de se sair bem no jogo. Como resultado dessa primeira etapa, tem-se a capacitação dos integrantes da equipe para ministrar minicursos em etapas subsequentes.

Na segunda etapa do Estudo de Caso, ocorreram visitas a escolas públicas e privadas de Ensino Médio da cidade de Patos de Minas / MG, para fins de divulgação e levantamento de estudantes interessados em participar do experimento. Estimulados pelo fato de o convite partir de alunos de sua faixa etária, os estudantes ficaram motivados a participar.

O minicurso de Robocode foi ministrado pelos alunos do projeto PIBIC-JR a alunos do Ensino Médio das escolas selecionadas, supervisionados pelos tutores. Alunos de graduação da UFU também foram convidados a participar. A Figura 5 ilustra uma foto do minicurso realizado em 2012.

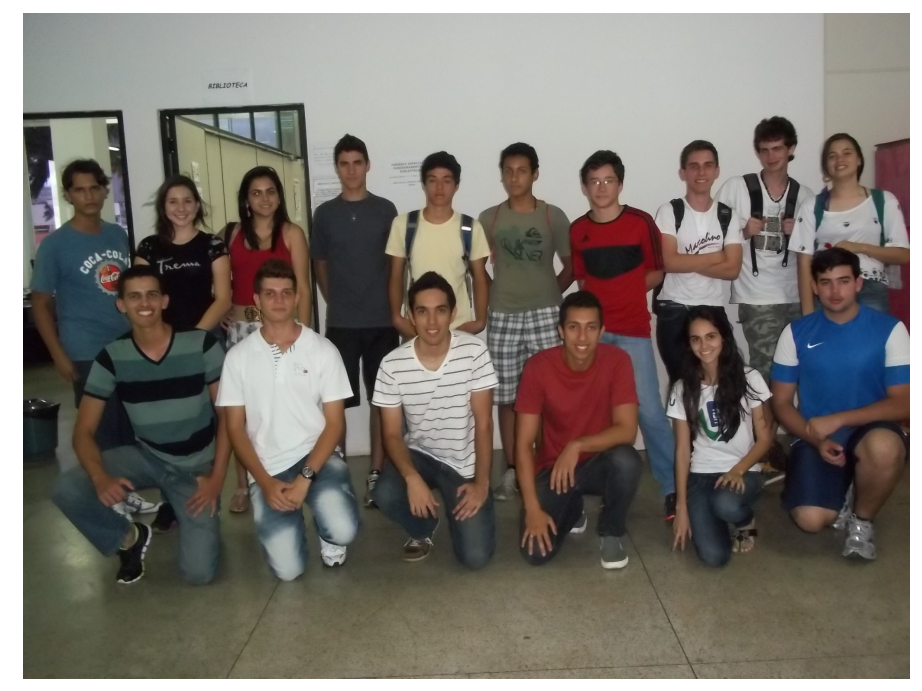

Figura 5. Participantes do minicurso de Robocode 2012

Após a realização dos minicursos, a terceira etapa envolveu a organização de um evento, voltado à realização de palestras e de uma competição baseada no ambiente Robocode. A organização do Torneio Robocode foi realizada pelos próprios alunos, com orientação dos professores envolvidos, e compreendeu as seguintes atividades:

- Criação das regras da competição;

- Busca de locais para realização do evento;

- Busca de patrocínios e recursos; e

- Divulgação do evento.

Foram realizadas três edições do evento (2012, 2013 e 2014). Os evento foram realizados com sucesso, consistindo de uma palestra ministrada pelos alunos de graduação, e da competição em si. Os três primeiros colocados foram premiados com vários brindes doados pelos patrocinadores. A Figura 6 apresenta os vencedores da competição nas três primeiras edições do evento. 


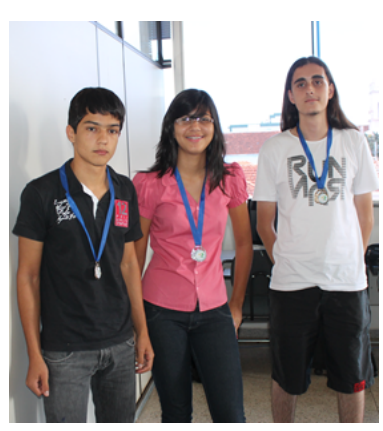

(a) 2012

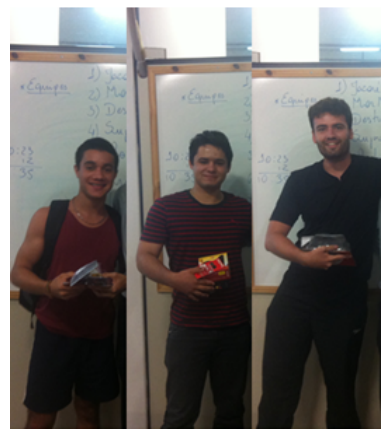

(b) 2013

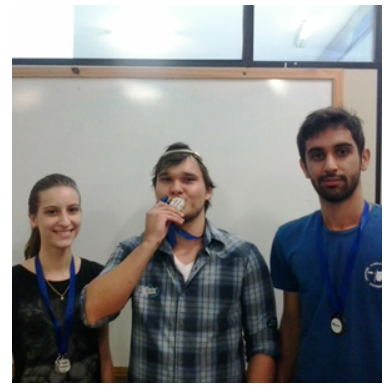

(c) 2014

Figura 6. Vencedores dos Torneios de Robocode

Por fim, foi realizada a divulgação dos resultados obtidos na mídia local, em relatórios e artigos técnicos e científicos, como forma de compartilhar a experiência obtida para toda a comunidade acadêmica e científica. Em 2012 e 2013, os alunos do Ensino Médio tiveram oportunidade de apresentar seu trabalho em uma mostra de ciência e tecnologia para alunos do Ensino Fundamental, Médio e Técnico [Santana et al. 2013].

\subsection{Análise e Discussão}

A partir desse Estudo de Caso, foi possível observar o potencial do ambiente Robocode como ferramenta lúdica facilitadora do processo de ensino-aprendizagem, aliado ao poder atrativo dos jogos no universo dos estudantes. Observou-se ainda que o uso do Robocode como ferramenta de ensino foi bem aceito também pelos próprios alunos da UFU / Patos de Minas envolvidos com o Estudo de Caso, podendo ser utilizado como auxiliador no ensino de algoritmos e linguagens de programação no âmbito da graduação.

Para a avaliação do minicurso ministrado pelos alunos do Ensino Médio, foi solicitado a cada participante que respondesse um questionário. A Tabela 1 mostra as perguntas realizadas, as alternativas disponíveis e as porcentagens de cada resposta, calculadas sobre o total de alunos nas três edições do projeto. Em geral, observa-se uma avaliação bastante positiva da metodologia.

No que compete aos bolsistas envolvidos, além de receberem treinamentos em algoritmos e na linguagem de programação Java, através do ambiente Robocode, a responsabilidade atribuída a eles proporcionou grande amadurecimento pessoal e profissional. Além de ministrar o minicurso, os alunos tiveram a oportunidade de participar da organização de um evento, praticando o trabalho em equipe e desenvolvendo seu senso de liderança, experiências que contribuem significativamente para seu desenvolvimento.

\section{Considerações Finais}

Com o desenvolvimento desse trabalho, percebeu-se, na prática, o quanto o uso de jogos educacionais tem se mostrado uma estratégia de ensino promissora. Acredita-se que a aprendizagem por meio da investigação venha a desenvolver o senso crítico dos alunos envolvidos, estimulando a busca pelo conhecimento e a competição. Com o Robocode, os alunos do Ensino Médio tiveram a oportunidade de desenvolver seu raciocínio lógico e aplicar conceitos básicos de Matemática em um ambiente lúdico de ensino, atrativo e interativo. 
CBIE-LACLO 2015

Anais do XXVI Simpósio Brasileiro de Informática na Educação (SBIE 2015)

Tabela 1. Avaliação do minicurso de Robocode

\begin{tabular}{|c|c|c|}
\hline Pergunta & Alternativas & Porcentagem \\
\hline Já conhecia a plataforma Robocode? & Sim & $3 \%$ \\
& Não & $90 \%$ \\
& Vagamente & $7 \%$ \\
\hline O mini-curso realizado proporcionou-me novos conhecimentos? & Sim & $100 \%$ \\
& Não & $0 \%$ \\
\hline Os métodos usados no treinamento pelo instrutores foram: & Adequados & $100 \%$ \\
& Pouco adequados & $0 \%$ \\
\hline O material didático utilizado foi: & Suficiente & $100 \%$ \\
& Insuficiente & $0 \%$ \\
\hline A qualidade (conteúdo) do material didático foi: & Muito Boa & $47 \%$ \\
& Boa & $53 \%$ \\
& Razoável & $0 \%$ \\
& Deficiente & $0 \%$ \\
\hline Avaliação dos instrutores (de 0 a 10): & Média & 9,51 \\
\hline O número de horas diárias do mini-curso foi: & Adequado & $91 \%$ \\
& Insuficiente & $7 \%$ \\
& Excessivo & $2 \%$ \\
\hline Classifico, de um modo geral, o mini-curso realizado como: & Excelente & $50 \%$ \\
& Muito bom & $41 \%$ \\
& Bom & $4 \%$ \\
& Regular & $3 \%$ \\
& Sofrível & $2 \%$ \\
\hline
\end{tabular}

Acredita-se ainda que a inserção da programação para estudantes do Ensino Médio constitua uma estratégia de captação de alunos para a universidade. Além de favorecer o raciocínio lógico dos estudantes, esse contato prematuro com algoritmos e programação pode prepará-los para cursar disciplinas, direta ou indiretamente relacionadas, em cursos de graduação que eles venham a cursar.

Atividades de pesquisa e extensão como essa mostram-se primordiais na busca pelo fortalecimento da tríade ensino-pesquisa-extensão, em especial, no campus da UFU / Patos de Minas, pela possiblidade de integração entre universidade e sociedade e também pelo potencial de captação de alunos.

Devido aos resultados positivos obtidos, o experimento está sendo aplicado no ano de 2015, com realização de novos treinamentos, minicursos e eventos. Até o momento, novos bolsistas já foram selecionados e os treinamentos já estão em andamento.

\section{Agradecimentos}

Os autores agradecem à FAPEMIG, CAPES e CNPq pelo apoio financeiro.

\section{Referências}

Astrachan, O. and Briggs, A. (2012). CS principles: The CS principles project. ACM Inroads, 3(2):38-42.

Batista Junior, E. P. and Santos Filho, J. V. d. (2011). Utilizando ambientes virtuais como ferramenta de auxílio no ensino de física em escolas públicas. In Anais do XXII SBIE - Simpósio Brasileiro de Informática na Educação. 
CBIE-LACLO 2015

Anais do XXVI Simpósio Brasileiro de Informática na Educação (SBIE 2015)

Carnegie Mellon University (2015). Alice project. http://www.alice.org/ - Acesso em: 22/05/2015.

de Oliveira, G. A. A., de Bettio, R. W., Rodarte, A. P. M., Braz, J. E., and Ferrari, F. B. (2014). Grubibots educacional: jogo para o ensino de algoritmos na educação básica. In Anais dos Workshops do Congresso Brasileiro de Informática na Educação.

Leite, L. S., Pocho, C. L., de Medeiros Aguiar, M., and Sampaio, M. N. (2003). Tecnologia educacional. Vozes, Petrópolis.

Maike, V. R. M. L., de Miranda, L. C., and Baranauskas, M. C. C. (2011). Relato de uma experiência no ensino de algoritmos e programação utilizando um framework lúdico. In Anais do XXII SBIE - Simpósio Brasileiro de Informática na Educação.

Pietruchinski, M. H., Neto, J. C., Malucelli, A., and Reinehr, S. (2011). Os jogos educativos no contexto do sbie: uma revisão sistemática de literatura. In Anais do XXII SBIE - Simpósio Brasileiro de Informática na Educação.

PUC-Rio/SERG (2013). Scalable game design brasil (sgd-br). http://www.sgd-br.inf.pucrio.br - Acesso em: 25/05/2015.

PUC-Rio/SERG (2015). Polifacets. http://www.serg.inf.puc-rio.br/polifacets/resources/ view_html_help.lua?lg=pt_br\&id_resource=81 - Acesso em: 25/05/2015.

Roberto G. Morati Jr., Alan P. Berger, O. d. L. T. C. S. d. M. (2012). Alice no labirinto das decisões: um jogo para exercitar a tomada de decisão e planejamento. In Anais dos Workshops do Congresso Brasileiro de Informática na Educação.

Santana, A. L. B., Guimarães, L. G. S., de Santana, E. M., and do Amaral, L. R. (2013). Utilização do ambiente robocode como estratégia para ensino de algoritmos e linguagem de programação java para alunos do ensino médio. In Anais da III MOCTI Mostra de Ciência e Tecnologia da cidade de Ituiutaba.

Santos, R. P. d. and Costa, H. A. X. (2006). Análise de metodologias e ambientes de ensino para algoritmos, estruturas de dados e programação aos iniciantes em computação e informática. Infocomp Journal of Computer Science, 5(1):41-50.

SourceForge (2015). Robocode home. http://robocode.sourceforge.net/ - Acesso em: 20/05/2015.

Vahldick, A. and Mattos, M. M. (2008). Relato de uma experiência no ensino de algoritmos e programação utilizando um framework lúdico. In Anais do XIX SBIE - Simpósio Brasileiro de Informática na Educação.

Viegas, M. A. C., Vieira, M. B., and de Souza da Silva, R. L. (2012). Ferramenta de apoio ao ensino de física utilizando realidade aumentada. Revista Brasileira de Informática na Educação, 20(3).

Washington University (2015). Ap computer science principles. https:// advancesinap.collegeboard.org/stem/computer-science-principles - Acesso em: 25/05/2015. 